\title{
Novel bHLH and WD40 transcription factors from turmeric (Curcuma longa L.) as putative regulators of curcumin biosynthesis
}

\author{
P. Prashina Mol, R.S. Aparna, T.E. Sheeja* and K. Deepa \\ ICAR-Indian Institute of Spices Research, Kozhikode-673 012, Kerala, India
}

(Manuscript Received: 11-12-2020, Revised: 31-01-2021, Accepted:15-02-2021)

\begin{abstract}
Turmeric, the golden spice belonging to the family Zingiberaceae, is enriched with biologically active curcuminoids composed of curcumin, demethoxycurcumin and bisdemethoxycurcumin. Curcuminoids are phenylpropanoid derivatives, and the biosynthetic pathway is controlled by several transcription factors (TFs). bHLH, WD40 and MYB TFs are the most important TFs regulating phenylpropanoid biosynthesis in plants. Through comparative transcriptome analysis of high and low curcumin germplasm accessions, 20 TFs belonging to the classes bHLH, WD 40, NAC, WRKY and bZIP, which showed differential expression with respect to curcumin, were identified. Among these, two bHLH and one WD40 TFs showed maximum comparative fold change and negative correlation vis-a-vis curcumin content in quantitative real-time polymerase chain reaction (qRTPCR) analysis. The results of comparative transcriptome and qRT-PCR analyses were in congruence, indicating their putative role as negative regulators.
\end{abstract}

Keywords: Curcumin biosynthetic pathway, qRT-PCR, transcription factors, transcriptome

\section{Introduction}

Curcuminoids are biologically active compounds present in rhizomes of turmeric (Curcuma longa L.). Curcuminoids are a mixture of curcumin, demethoxycurcumin and bisdemethoxycurcumin. In recent years curcumin has attracted significant attention due to its health-promoting effects, such as protection against cancer, heart diseases (Wongcharoen and Phrommintikul, 2009) and other chronic human disorders (Mantzorou et al., 2018). Moreover, curcumin has also been used as a natural replacement for synthetic food colourants (Ismail and Sakr, 2016) and is an integral part of religious ceremonies (Chattopadhyay et al., 2004).

Curcuminoids are a class of secondary metabolites synthesised through complex biosynthetic pathways that involve structural genes responsible for the formation of final metabolite and regulatory genes that regulate their expression.
Curcuminoid biosynthesis involves several enzymecoding structural genes such as phenylalanine ammonia lyase $(P A L)$, cinnamate 4 hydroxylase $(\mathrm{C} 4 \mathrm{H}), 4$-coumaryl CoA ligase $(4 \mathrm{CL})$, diketide CoA synthase (DCS) and curcumin synthases 1,2, and 3 (CURS1, CURS2, CURS3) (Katsuyama et al., 2009a; 2009b). Earlier studies from our lab have reported certain novel structural genes like CLPKS11 with a putative role in biosynthesis (Deepa et al., 2017). The expression of these structural genes is regulated at the transcriptional level by TFs. TFs are sequencespecific DNA-binding proteins that interact with the regulatory regions of the target genes and control transcriptional initiation rate (Vom Endt et al., 2002). These regulators bind to homologous cis-elements situated in promoters and regulate the expression of biosynthetic pathway genes or interact with other TFs to form a complex (Yang et al., 2012, Zhao et al., 2019). Several families of TFs have been described as

*Corresponding Author: sheejate@icar.gov.in 
regulators of plant secondary metabolism that include bHLH, WD40, MYB, WRKY, bZIP, NAC etc. (Zhao et al., 2013; Saga et al., 2012; Xu et al., 2004; Wang et al., 2015). Biosynthesis of phenylpropanoids is generally controlled by a gene regulatory network involving MYB transcription factors regulating early biosynthetic steps and a complex of MYBbHLH-WD40 TFs regulating the late biosynthetic steps (Li et al., 2014). Late biosynthetic steps are more specific to the final metabolite and are more important for pathway engineering. We have already reported MYB TFs regulating curcumin biosynthesis in an earlier study (Sheeja et al., 2015); however, no information on key TFs such as bHLH and WD40 are available. Hence, in the present study, we evaluated bHLH and WD40 TFs and identified three novel TFs that negatively regulate curcumin biosynthesis, which may lead to unravelling the regulatory mechanism.

\section{Materials and methods}

\section{Plant material}

Rhizomes were collected after 120 days of planting from high-curcumin turmeric- IISR Prathibha, low curcumin turmeric- Accession No. 200 and a closely related species that is practically devoid of curcumin- $C$. aromatica from the Experimental Farm, Chelavoor of ICAR-Indian Institute of Spices Research (ICAR-IISR), Kozhikode, Kerala State, India (Table 1).

\section{Screening of Curcuma transcriptome for different transcription factor unigenes}

Homologous transcripts of different types of TFs like bHLH, WD40, NAC, WRKY, bZIP with fold change $>3$ was retrieved from the text of annotated genes within transcriptome data of C. longa (high curcumin) and C. aromatica (practically devoid of curcumin) (Sheeja et al., 2015) (Table 2). Gene ontology (GO) terms were retrieved for each unigene using the default settings of Blast 2GO software (Conesa and Gotz, 2008).

\section{Quantitative real-time PCR}

Total RNA was isolated as per Deepa et al. (2014), and three biological replicates were pooled for each sample and were digested using an oncolumn DNase I digestion set. The quality and quantity of RNA were checked by agarose gel electrophoresis (1.2\%) and Biophotometer plus (Eppendorf, Germany). The RNA samples showed two discrete bands of $28 \mathrm{~S}$ and $18 \mathrm{~S}$ rRNA on agarose gel and A260/280 ratio between 1.8 and 2.0. About $250 \mathrm{ng}$ of total RNA was used for first-strand cDNA synthesis using Superscript III reverse transcriptase (Invitrogen) and Oligo-(dT)18 primer in a total volume of $20 \mu \mathrm{L}$. Gene expression profiling was performed on Rotor-Gene Q (Qiagen) using the QuantiFast SYBR Green PCR kit (Qiagen). The reaction mixture comprised of $10 \mu \mathrm{L}$ of $2 \mathrm{X}$ SYBR Green, 10 pmol each of gene-specific primers and $100 \mathrm{ng}$ of cDNA in a final volume of $20 \mu \mathrm{L}$. qRT-PCR amplification was performed under the following conditions: $95^{\circ} \mathrm{C}$ for $5 \mathrm{~min}$, followed by 40 cycles of $95^{\circ} \mathrm{C}$ for $10 \mathrm{~s}$ and $60^{\circ} \mathrm{C}$ for $30 \mathrm{sec}$. A melt curve program of $65-99^{\circ} \mathrm{C}$ was included to check the specificity of qRT-PCR products. Three technical replicates per reaction were performed, and the mean $\mathrm{Ct}$ value was used for the analysis. A reverse transcription negative control (without reverse transcriptase) and a non-template negative control were included to confirm the absence of genomic DNA and check non-specific amplification. The primers for $20 \mathrm{TFs}$ were designed based on previous transcriptome data (Sheeja et al., 2015) using Primer Quest (www.idtdna.com/primer quest/ home/index); the details of the primers are listed in Table 2. Candidate reference genes viz., EFl $\alpha$ (FP-GCTGACTGTGCTGTTCTCATTAT and RP-CTCGTGTCTGTCCATCCTTTGAA) and

Table 1. Details of Curcuma species chosen for the study and curcumin contents

\begin{tabular}{lll}
\hline Curcuma species & Source & Curcumin content (\%) \\
\hline IISR Prathibha & ICAR-IISR germplasm & 5.37 (high) \\
Accession No. 200 & ICAR-IISR germplasm & 3.54 (low) \\
C. aromatica & ICAR-IISR germplasm & 0.12 (very low) \\
\hline
\end{tabular}




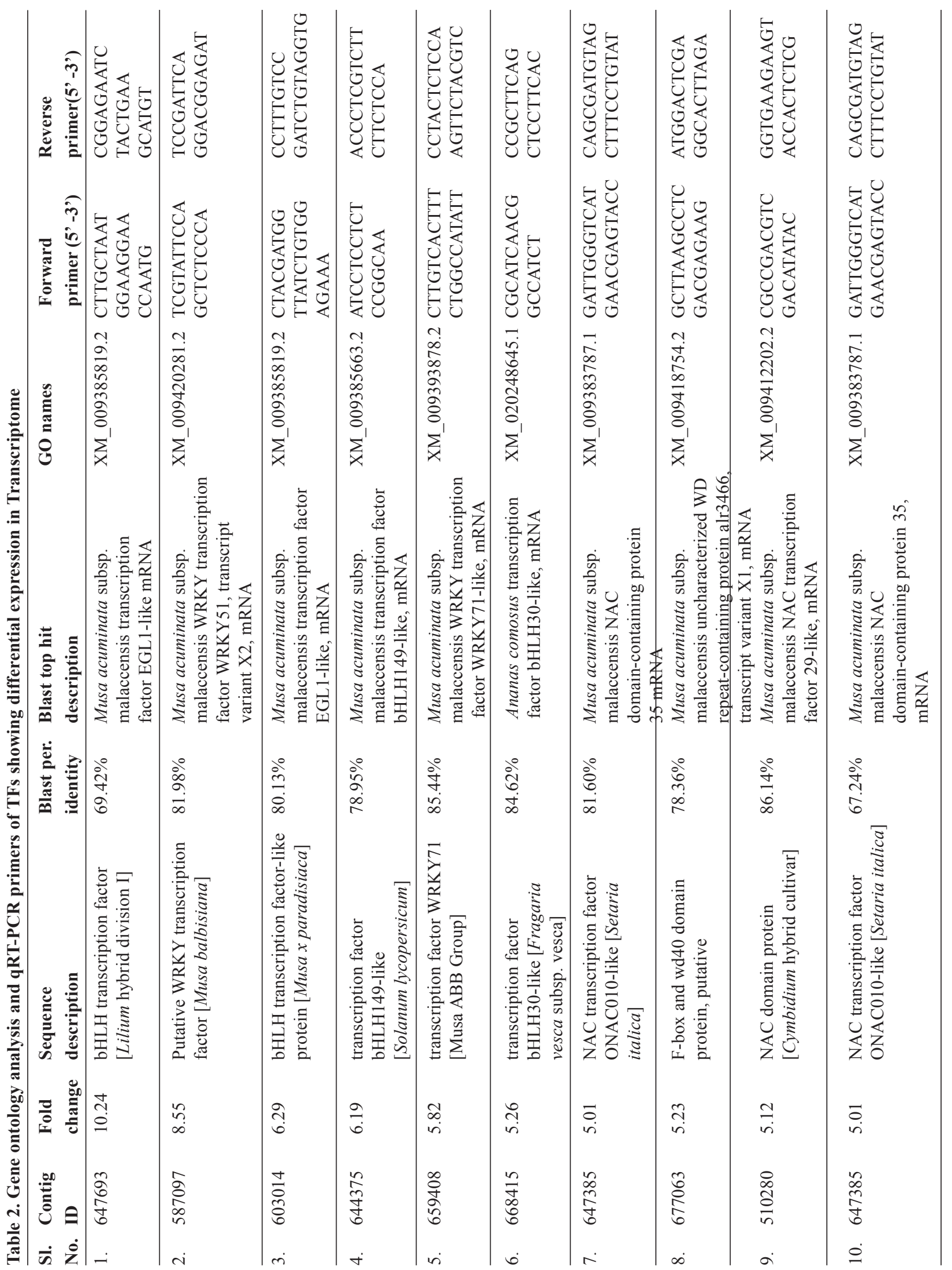




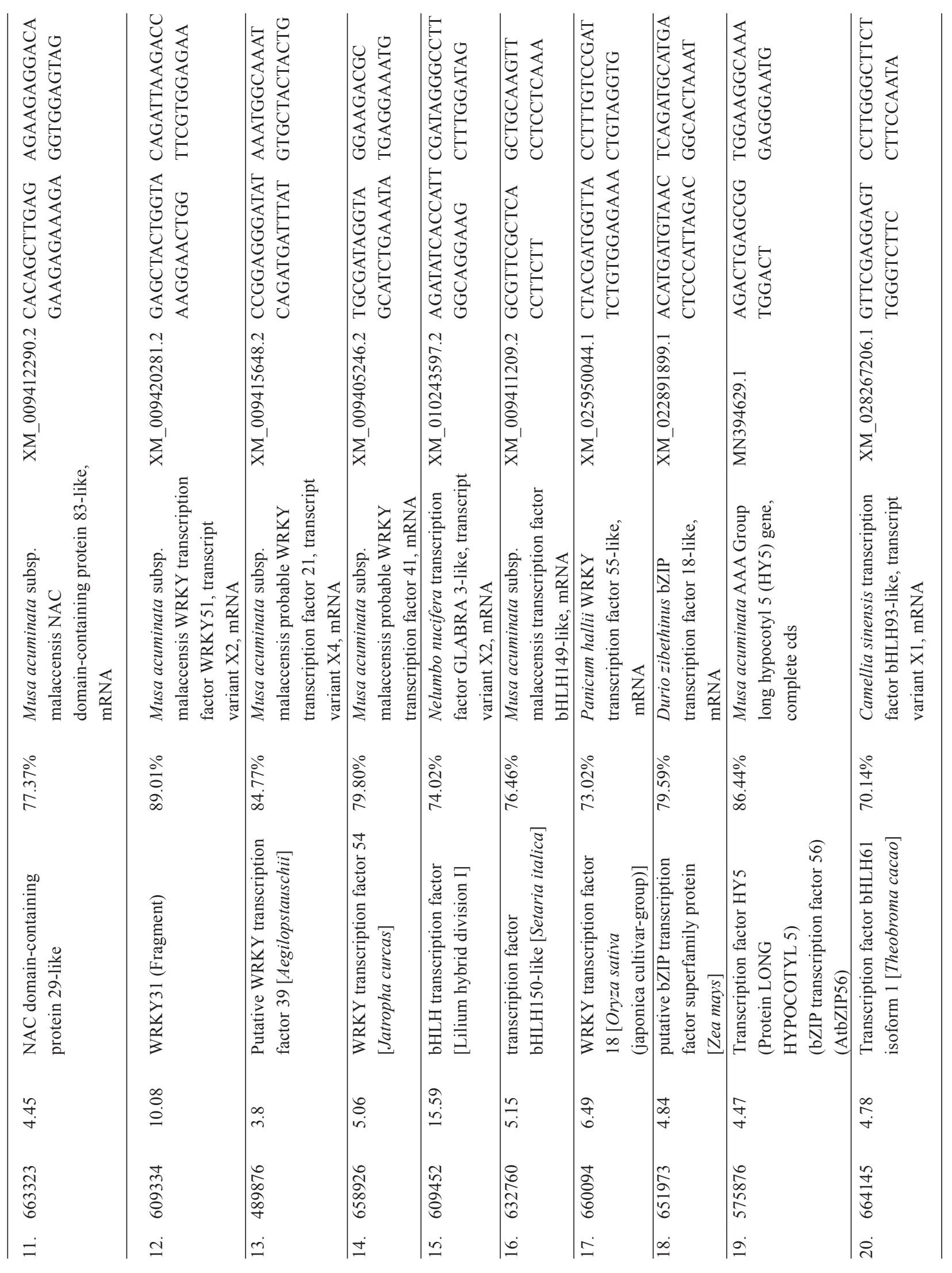


Ubiquitin (FP-GCACTCTCGCTGACTACAAC and RP- GGCTTGGTGTAGGTCTTCTTC) were used as per Deepa et al. (2017). Standard curves were generated for each gene with serial dilutions of pooled cDNAs $\left(10^{-1}\right.$ to $\left.10^{-6}\right)$ for calculating qRT-PCR efficiency. The relative gene expression was calculated using the $2^{-\Delta \Delta C t}$ method (Livak and Schmittgen 2001) and transformed to a $\log 2$ scale.

\section{Estimation of curcumin content}

Curcumin content of rhizome samples was analysed spectrophotometrically at $430 \mathrm{~nm}$ following the American Spice Trade Association (1968) procedure (Table 1).

\section{Results and discussion}

\section{Identification of TFs from Curcuma transcriptome}

Transcriptome analysis is widely reported as an effective method for identifying regulatory molecules like TFs in many studies involving phenylpropanoids (Zong et al., 2019; Song et al., 2019; Todd et al., 2010). Aligning the annotated transcripts of $C$. longa and $C$. aromatica to the AGRIS database resulted in the identification of unigenes belonging to 39 transcription factor families in C. longa and 36 transcription factor families in $C$. aromatica, which included bHLH, MYB, WRKY, MADS, NAC, bZIP, ARR-B, C2H2 and AP2-EREBP TFs (Sheeja et al., 2015). Among these, seven bHLH (contig ID nos. 647693, 603014, 644375, 644375, 668415, 609452, 632760), six WRKY (contig ID nos. 587097, 659408, 658926, 660094, 609334, 489876), three NAC (contig ID nos. 647385, 510280, 663323), two bZIP (contig ID nos. 651973, 575876) and one WD40 (contig ID no. 677063) showed maximum fold change vis-a-vis curcumin levels. Several families of TFs have been described to be regulators of plant secondary metabolism, which include bHLH (Hong et al., 2012; Shoji et al., 2011; Zhang et al., 2011; Todd et al., 2010), WD40 (Li et al., 2014), MYB (Teng et al., 2005, Gonzalez et al., 2008; Yamagishi et al., 2010; Butelli et al., 2012; Xu et al., 2017), WRKY, (Suttipanta et al., 2011; Ma et al., 2009), bZIP, (Yu et al., 2012), NAC (Saga et al., 2012) etc.
Biosynthesis of phenylpropanoid derivatives like flavonoids is generally controlled by MYB TFs and MYB-bHLH-WD40(MBW) complexes (Schaart et al., 2013; Li et al., 2014; Xu et al., 2015).

\section{qRT-PCR expression patterns of TFs}

qRT-PCR analysis is a common strategy to validate the RNA-Seq data. Twenty shortlisted TFs from transcriptome analysis were further evaluated by qRT-PCR with IISR Prathibha as control. Among these contig IDs 603014 and 668415 (bHLH TFs) and contig ID 677063 (WD40 TF) showed the highest relative fold change with respect to curcumin (low $v s$ high) (Fig. 1). The expression of ClPKS11, a bait gene that is found to be upregulated under high curcumin conditions (Deepa et al., 2017), was high in IISR Prathibha and down-regulated in low curcumin accessions of $C$. aromatica and Accession No. 200. bHLH TFs (Contig IDs 603014 and 668415) and WD40 TF (contig ID 677063) showed a negative correlation with respect to the expression of ClPKS11 (Fig. 1). If one or more genes in a biosynthetic pathway for a particular metabolite is identified, these genes may be used as baits to identify other novel candidates involved in the pathway through a co-expression analysis (Medema and Osbourn, 2016). Several studies have reported that bHLH members play a central role in transcriptional regulation of biosynthetic pathways by forming a complex with WD40 and MYB TF proteins (Zhao et al., 2019). In flavonoid biosynthesis, additional regulators which interact with bHLHs or R2R3-MYBs to organize or disrupt the formation of the MBW complex, leading to enhanced or compromised flavonoid production, have been reported (Li et al., 2014). The qRT-PCR trends were in congruence with the fold change values in RNA-seq Data. Thus, we have identified three novel TFs belonging to the bHLH and WD40 classes to add up to the several MYB TFs identified in our earlier studies (Sheeja et al., 2015). Blastn search indicated the unique status of all three TFs based on the per cent identity (Table 2). These novel TFs, in conjunction with the already identified MYB TFs will be instrumental in future studies to confirm the mechanism of the molecular regulation of curcumin biosynthesis. 


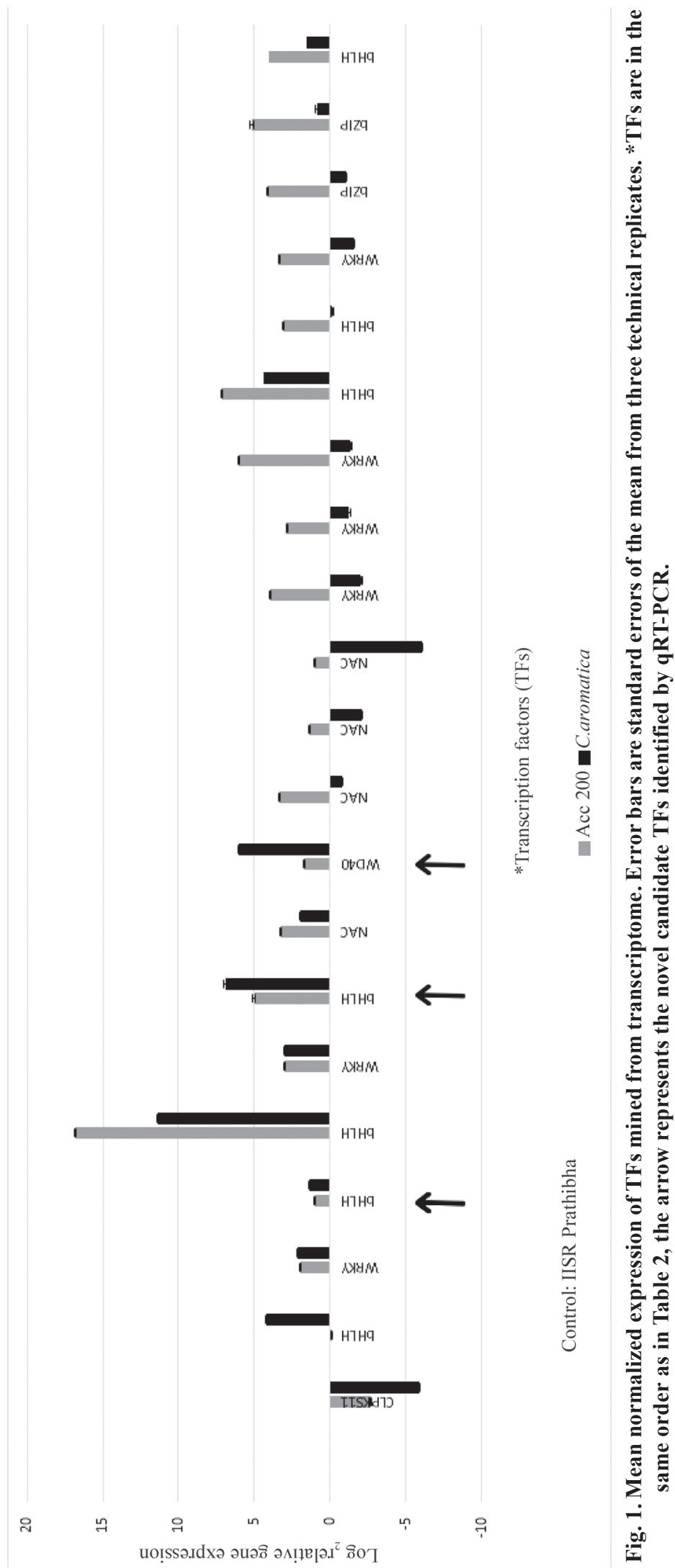




\section{Conclusion}

Curcumin is a phenylpropanoid derivative, and its biosynthesis is regulated by several classes of TFs, with bHLH, WD40 and MYB TFs being the most important. In the present study, we have identified 20 TFs through comparative transcriptome analysis. Among these, real-time expression patterns of two TFs belonging to bHLH and one belonging to WD40 categories indicated the highest differential fold change between high and low curcumin accessions and a negative correlation with curcumin content. Their expression was also negatively correlated with a bait gene CLPKS 11 in both transcriptome analysis and qRT-PCR. These novel TFs may help understand the molecular regulation of biosynthesis of curcumin, which is essential for developing high curcumin lines in turmeric.

\section{Acknowledgements}

The first author is thankful to the University Grants Commission (UGC), New Delhi, for the fellowship. The authors are grateful to the Director, ICAR-Indian Institute of Spices Research, for providing the facilities.

\section{References}

Butelli, E., Licciardello, C., Zhang, Y., Liu, J., Mackay, S., Bailey, P. and Martin, C. 2012. Retrotransposons control fruit-specific, cold-dependent accumulation of anthocyanins in blood oranges. The Plant Cell 24(3): 1242-1255.

Chattopadhyay, I., Biswas, K., Bandyopadhyay, U. and Banerjee, R. K. 2004. Turmeric and curcumin: Biological actions and medicinal applications. Current Science 87: 44-53.

Conesa, A. and Gotz, S. 2008. Blast2GO: A comprehensive suite for functional analysis in plant genomics. International Journal of Plant Genomics 1-12; doi: $10.1155 / 2008 / 619832$.

Deepa, K., Sheeja, T. E., Rosana, O. B., Srinivasan, V., Krishnamurthy, K. S., and Sasikumar, B. 2017. Highly conserved sequence of ClPKS11 encodes a novel polyketide synthase involved in curcumin biosynthesis in turmeric (Curcuma longa L.). Industrial Crops and Products 97: 229-241.

Deepa, K., Sheeja, T. E., Santhi, R., Sasikumar, B., Cyriac, A., Deepesh, P. V. and Prasath, D. 2014. A simple and efficient protocol for isolation of high-quality functional RNA from different tissues of turmeric (Curcuma longa L.).
Physiology and Molecular Biology of Plants 20(2): 263-271.

Gonzalez, A., Zhao, M., Leavitt, J. M. and Lloyd, A. M. 2008. Regulation of the anthocyanin biosynthetic pathway by the TTG1/bHLH/Myb transcriptional complex in Arabidopsis seedlings. The Plant Journal 53(5): 814-827.

Hong, G. J., Xue, X. Y., Mao, Y. B., Wang, L. J. and Chen, X. Y. 2012. Arabidopsis MYC2 interacts with DELLA proteins in regulating sesquiterpene synthase gene expression. The Plant Cell 24(6): 2635-2648.

Ismail, M., and Sakr, S. 2016. Validation of replacement of the synthetic food dye 'sunset yellow'-induced hepatotoxicity and genotoxicity with the nutraceutical 'curcumin' in mice. Merit Research Journals of Medicine and Medical Sciences 4(1): 25-50.

Katsuyama, Y., Kita, T. and Horinouchi, S. 2009a. Identification and characterization of multiple curcumin synthases from the herb Curcuma longa. FEBS letters 583(17): 2799-2803.

Katsuyama, Y., Kita, T., Funa, N., and Horinouchi, S. 2009b. Curcuminoid biosynthesis by two type III polyketide synthases in the herb Curcuma longa. Journal of Biological Chemistry 284(17): 11160-11170.

Li, S. 2014. Transcriptional control of flavonoid biosynthesis: fine-tuning of the MYB-bHLH-WD40 (MBW) complex. Plant Signaling and Behavior 9(1): 27522.

Livak, K. J. and Schmittgen, T. D. 2001. Analysis of relative gene expression data using real-time quantitative PCR and the $2^{-\Delta \Delta C t}$ method. Methods 25(4): 402-408.

Ma, D., Pu, G., Lei, C., Ma, L., Wang, H., Guo, Y., and Ye, H. 2009. Isolation and characterization of AaWRKY1, an Artemisia annua transcription factor that regulates the amorpha-4, 11-diene synthase gene, a key gene of artemisinin biosynthesis. Plant and Cell Physiology 50(12): 2146-2161.

Mantzorou, M., Pavlidou, E., Vasios, G., Tsagalioti, E. and Giaginis, C. 2018. Effects of curcumin consumption on human chronic diseases: A narrative review of the most recent clinical data. Phytotherapy Research 32(6): 957-975.

Medema, M. H. and Osbourn, A. 2016. Computational genomic identification and functional reconstitution of plant natural product biosynthetic pathways. Natural Product Reports 33(8): 951-962.

Saga, H., Ogawa, T., Kai, K., Suzuki, H., Ogata, Y., Sakurai, N. and Ohta, D. 2012. Identification and characterization of ANAC042, a transcription factor family gene involved in the regulation of camalexin biosynthesis in Arabidopsis. Molecular Plant-Microbe Interactions 25(5): 684-696.

Schaart, J. G., Dubos, C., Romero De La Fuente, I., van Houwelingen, A. M., de Vos, R. C., Jonker, H. H. and 
Bovy, A. G. 2013. Identification and characterization of MYB bHLH WD40 regulatory complexes controlling proanthocyanidin biosynthesis in strawberry (Fragaria x ananassa) fruits. New Phytologist 197(2): 454-467.

Sheeja, T. E., Deepa, K., Santhi, R. and Sasikumar, B. 2015. Comparative transcriptome analysis of two species of Curcuma contrasting in a high-value compound curcumin: insights into genetic basis and regulation of biosynthesis. Plant Molecular Biology Reporter 33(6): 1825-1836.

Shoji, T. and Hashimoto, T. 2011. Tobacco MYC2 regulates jasmonate-inducible nicotine biosynthesis genes directly and by way of the NIC2-locus ERF genes. Plant and Cell Physiology 52(6): 1117-1130.

Song, T., Li, K., Wu, T., Wang, Y., Zhang, X., Xu, X. and Han, Z. 2019. Identification of new regulators through transcriptome analysis that regulate anthocyanin biosynthesis in apple leaves at low temperatures. PloS one 14(1): e0210672.

Suttipanta, N., Pattanaik, S., Kulshrestha, M., Patra, B., Singh, S. K. and Yuan, L. 2011. The transcription factor CrWRKY1 positively regulates the terpenoid indole alkaloid biosynthesis in Catharanthus roseus. Plant Physiology 157(4): 2081-2093.

Teng, S., Keurentjes, J., Bentsink, L., Koornneef, M. and Smeekens, S. 2005. Sucrose-specific induction of anthocyanin biosynthesis in Arabidopsis requires the MYB75/PAP1 gene. Plant Physiology 139(4): 1840-1852.

Todd, A. T., Liu, E., Polvi, S. L., Pammett, R. T. and Page, J. E. 2010. A functional genomics screen identifies diverse transcription factors that regulate alkaloid biosynthesis in Nicotiana benthamiana. The Plant Journal 62(4): 589-600.

Vom Endt, D., Kijne, J. W., and Memelink, J. 2002. Transcription factors controlling plant secondary metabolism: what regulates the regulators? Phytochemistry 61(2): 107-114.

Wang, X., Wu, F., Liu, L., Liu, X., Che, Y., Keller, N. P. and Yin, W. B. 2015. The bZIP transcription factor PfZipA regulates secondary metabolism and oxidative stress response in the plant endophytic fungus Pestalotiopsis fici. Fungal Genetics and Biology 81: 221-228.

Wongcharoen, W. and Phrommintikul, A. 2009. The protective role of curcumin in cardiovascular diseases. International Journal of Cardiology 133(2): 145-151.
Xu, W., Dubos, C., and Lepiniec, L. 2015. Transcriptional control of flavonoid biosynthesis by MYB-bHLH-WDR complexes. Trends in Plant Science 20(3): 176-185.

Xu, Y. H., Wang, J. W., Wang, S., Wang, J. Y. and Chen, X. Y. 2004. Characterization of GaWRKY1, a cotton transcription factor that regulates the sesquiterpene synthase gene (+)-ä-cadinene synthase-A. Plant Physiology 135(1): 507-515.

Xu, Z. S., Feng, K., Que, F., Wang, F. and Xiong, A. S. 2017. A MYB transcription factor, DcMYB6, is involved in regulating anthocyanin biosynthesis in purple carrot taproots. Scientific Reports 7: 45324.

Yamagishi, M., Shimoyamada, Y., Nakatsuka, T., and Masuda, K. 2010. Two R2R3-MYB genes, homologs of petunia AN2, regulate anthocyanin biosyntheses in flower tepals, tepal spots and leaves of Asiatic hybrid lily. Plant and Cell Physiology 51(3): 463-474.

Yang, C. Q., Fang, X., Wu, X. M., Mao, Y. B., Wang, L. J. and Chen, X. Y. 2012. Transcriptional regulation of plant secondary metabolism F. Journal of Integrative Plant Biology 54(10): 703-712.

Yu, Z. X., Li, J. X., Yang, C. Q., Hu, W. L., Wang, L. J. and Chen, X. Y. 2012. The jasmonate-responsive AP2/ERF transcription factors AaERF1 and AaERF2 positively regulate artemisinin biosynthesis in Artemisia annua L. Molecular Plant 5(2): 353-365.

Zhang, H., Hedhili, S., Montiel, G., Zhang, Y., Chatel, G., Pre, M. and Memelink, J. 2011. The basic helix loop helix transcription factor CrMYC2 controls the jasmonate responsive expression of the ORCA genes that regulate alkaloid biosynthesis in Catharanthus roseus. The Plant Journal 67(1): 61-71.

Zhao, L., Gao, L., Wang, H., Chen, X., Wang, Y., Yang, H. and Xia, T. 2013. The R2R3-MYB, bHLH, WD40, and related transcription factors in flavonoid biosynthesis. Functional and Integrative Genomic 13(1): 75-98.

Zhao, M., Li, J., Zhu, L., Chang, P., Li, L. and Zhang, L. 2019. Identification and characterization of MYB-bHLHWD40 regulatory complex members controlling anthocyanidin biosynthesis in blueberry fruits development Genes 10(7): 496.

Zong, Y., Li, S., Xi, X., Cao, D., Wang, Z., Wang, R. and Liu, B. 2019. Comprehensive influences of overexpression of a MYB transcriptor regulating anthocyanin biosynthesis on transcriptome and metabolome of tobacco leaves. International Journal of Molecular Sciences 20(20): 5123. 